\title{
Native T1 mapping: inter-study, inter-observer and inter-center reproducibility in hemodialysis patients
}

\author{
Matthew P. M. Graham-Brown ${ }^{1,2,3^{*}}$, Elaine Rutherford ${ }^{4,5}$, E. Levelt ${ }^{6}$, Daniel S. March ${ }^{1,2}$, Darren R. Churchward ${ }^{1,2}$, \\ David J. Stensel ${ }^{3}$, Christie McComb ${ }^{4,7}$, Kenneth Mangion ${ }^{4,8}$, Samantha Cockburn ${ }^{4}$, Colin Berry ${ }^{4,8}$, James C. Moon ${ }^{9}$, \\ Patrick B. Mark ${ }^{4,5}$, James O. Burton ${ }^{1,2,6}$ and Gerry P. McCann ${ }^{6}$
}

\begin{abstract}
Background: Native T1 mapping is a cardiovascular magnetic resonance (CMR) technique that associates with markers of fibrosis and strain in hemodialysis patients. The reproducibility of $\mathrm{T} 1$ mapping in hemodialysis patients, prone to changes in fluid status, is unknown. Accurate quantification of myocardial fibrosis in this population has prognostic potential.

Methods: Using 3 Tesla CMR, we report the results of 1) the inter-study, inter-observer and intra-observer reproducibility of native T1 mapping in 10 hemodialysis patients; 2 ) inter-study reproducibility of left ventricular (LV) structure and function in 10 hemodialysis patients; 3 ) the agreement of native T1 map and native T1 phantom analyses between two centres in 20 hemodialysis patients; 4) the effect of changes in markers of fluid status on native T1 values in 10 hemodialysis patients.
\end{abstract}

Results: Inter-study, inter-observer and intra-observer variability of native T1 mapping were excellent with co-efficients of variation (CoV) of 0.7, 0.3 and $0.4 \%$ respectively. Inter-study CoV for LV structure and function were: LV mass = 1\%; ejection fraction $=1.1 \%$; LV end-diastolic volume $=5.2 \%$; LV end-systolic volume $=5.6 \%$. Inter-centre variability of analysis techniques were excellent with CoV for basal and mid-native T1 slices between 0.8-1.2\%. Phantom analyses showed comparable native T1 times between centres, despite different scanners and acquisition sequences (centre 1: $1192.7 \pm 7$. $5 \mathrm{~ms}$, centre 2: $1205.5 \pm 5 \mathrm{~ms}$ ). For the 10 patients who underwent inter-study testing, change in body weight ( $\Delta$ weight) between scans correlated with change in LV end-diastolic volume ( $\Delta$ LVEDV $(r=0.682 ; P=0.03)$ representing altered fluid status between scans. There were no correlations between change in native T1 between scans $(\Delta T 1)$ and $\Delta L V E D V$ or $\Delta$ weight $(P>0.6)$. Linear regression confirmed $\Delta T 1$ was unaffected by $\Delta$ LVEDV or $\Delta$ weight $(P>0.59)$.

Conclusions: Myocardial native $\mathrm{T1}$ is reproducible in $\mathrm{HD}$ patients and unaffected by changes in fluid status at the levels we observed. Native 11 mapping is a potential imaging biomarker for myocardial fibrosis in patients with end-stage renal disease.

Keywords: Hemodialysis, Myocardial fibrosis, Native T1, Reproducibility, Cardiovascular magnetic resonance

\section{Background}

There is an increased risk of cardiovascular (CV) mortality in chronic kidney disease (CKD), and end stage renal disease (ESRD) populations [1]. This increased risk can be attributed to the stereotyped changes that lead to the development of uremic cardiomyopathy and include, left

\footnotetext{
* Correspondence: mpmgb1@le.ac.uk

${ }^{1}$ John Walls Renal Unit, University Hospitals Leicester NHS Trust, Leicester, UK ${ }^{2}$ Department of Infection Immunity and Inflammation, School of Medicine and Biological Sciences, University of Leicester, Leicester LE1 9HN, UK

Full list of author information is available at the end of the article
}

ventricular (LV) hypertrophy, LV dilatation and myocardial fibrosis within the extracellular matrix [2]. To date, LV mass is the most commonly used surrogate end-point of mortality in clinical trials [3], as observational studies of $\mathrm{HD}$ patients have shown $\mathrm{LV}$ mass is good predictor of $\mathrm{CV}$ outcomes [4]. However, a recent systematic review and meta-analysis in patients of all stages of CKD suggested that there is no clear association between intervention-induced LV mass reduction and mortality [5]. Novel imaging biomarkers that can robustly and reliably measure pathological $\mathrm{CV}$ changes that link strongly to outcomes are required. 
Post-mortem studies of patients with CKD and ESRD on hemodiaysis (HD) demonstrate that uremia is a highly significant, independent determinant of extent of myocardial fibrosis [6]. Furthermore endomyocardial biopsy studies have shown that extent of myocardial fibrosis is the only independent predictor of death (mean follow-up period 3.1 years) for these patents [7]. As degree of myocardial fibrosis is the strongest predictor of increased CV mortality, defining a reliable measure of myocardial fibrosis in $\mathrm{HD}$ patients is a priority.

Cardiovascular Magnetic Resonance (CMR) with Late Gadolinium Enhancement (LGE) is an imaging biomarker used to assess myocardial fibrosis in many populations. Whilst gadolinium based contrast agents have previously been used to assess cardiac disease in HD patients [8] this is no longer possible due to the rare, but serious complication of nephrogenic systemic fibrosis [9]. Moreover, whilst LGE is a sensitive and reproducible way of assessing focal myocardial fibrosis, there are limitations in using gadolinium to assess diffuse myocardial fibrosis due to the reliance of the technique on demonstrating a difference between signal intensity of normal and fibrotic myocardial tissue [10]. Native T1 mapping is a novel, non-contrast CMR technique that correlates well with biopsy measured myocardial fibrosis in aortic stenosis [11, 12] and can differentiate patients with hypertrophic cardiomyopathy from hypertensive cardiac disease [13]. The inter-study repeatability and inter-observer variability of native T1 mapping has been shown to be very good in patients with aortic stenosis [14], patients with LV hypertrophy or dilated cardiomyopathy [15] and patients with Anderson-Fabry disease [16]. Myocardial native T1 times have been shown to be significantly higher in HD patients compared to control subjects $[17,18]$ and to associate with circulating markers of cardiac disease [17] and measures of myocardial systolic strain [18], but the reproducibility of native T1 mapping has not been assessed in HD patients who are prone to shifts in extracellular volume. Native T1 times are prolonged with increasing water content of tissue and the presence of intermittent myocardial edema from alterations in fluid status may in theory affect native T1 time confounding results and reducing native $\mathrm{T} 1$ time reproducibility. Concerns remain about the use of this technique to assess myocardial fibrosis in patients with ESRD on HD, who are subject to significant changes in fluid status and who may potentially have intermittent myocardial oedema [19].

In this study we aim to assess the reproducibility and reliability of native $\mathrm{T} 1$ mapping in patients with ESRD on HD by assessing: i) the inter-study, inter-observer and intra-observer variability of native T1 times in HD patients at 3 Tesla ( $3 \mathrm{~T})$; ii) the associations between changes in markers of fluid status and changes in native $\mathrm{T} 1$ times to assess the effect of fluid status on native T1 times; iii) the inter-centre reproducibility of native $\mathrm{T} 1$ values between two UK cardio-renal imaging centres, comparing analysis techniques of native T1 maps and native T1 values with 'phantom' analysis [20].

\section{Methods}

Patients from centre 1 were recruited as a part of the CYCYLE-HD study (ISRCTN 11299707) [21]. The study was given ethical approval by the NHS Research Ethics Committee East Midlands (Northampton; REC ref: 14/EM/ 1190). Patients from centre 2 were recruited as part of the observational cardiac uraemic fibrosis detection in dialysis patients study (CUDDLE study ISCRTN99591655). The study was approved by the West of Scotland Ethics committee (WoS 13/WS/0301). All participants gave written and informed consent.

\section{Inter-study reproducibility, inter-observer and intra-observer variability (centre 1 )}

Ten patients underwent a repeat CMR scan within 2 weeks of their initial scan. Patients receive dialysis three times per week on either a Monday, Wednesday, Friday, or a Tuesday, Thursday, Saturday. This means that there is a two-day break between dialysis once a week, commonly referred to as 'the long break'. Study patients were all scanned on a non-dialysis day within $24 \mathrm{~h}$ after their last dialysis and never during the long-break. The same CMR protocol was used for each scan (see below). We report the inter-study reproducibility of LV volumes, mass and of the mid-ventricular native $\mathrm{T} 1$ parametric mapping. Analysis of inter-study scans was conducted by a single, blinded, observer. Inter-observer variability of was conducted by 2 blinded observers on 10 native T1 maps independently from one another. Intra-observer variability was conducted by a single blinded observer.

\section{Inter-centre reproducibility (centre 1 and centre 2)}

The inter-observer variability of native $\mathrm{T} 1$ map analysis techniques between 2 UK cardio-renal imaging centres was assessed. The CMR scanners, native T1 mapping sequences and analysis techniques of centre 1 and centre 2 were different (see below). Ten basal and 10 mid-ventricular native $\mathrm{T} 1$ scans acquired at centre 1 were analysed by a blinded observer at centre 2, using the analysis technique of centre 2 and compared to the results of the analysis of the same scans analysed at centre 1 . Conversely 10 basal and mid-ventricular native T1 scans acquired at centre 2 were analysed by a blinded observer at centre 1 , using the analysis technique of centre 1 and compared to the results of the analysis of the same scans analysed at centre 2 . We also undertook native $\mathrm{T} 1$ phantom analysis at both centres. Phantom analyses were undertaken at centre 1 and centre 2 as part of the international, multi-centre T1MES project [20]. Phantom images recorded at a heart rate of approximately 70 beats per minute were obtained and compared. The phantoms were scanned once at each centre and the 
images were analysed at centre 2 . Uniform regions of interest within each relevant phantom area were determined using semi-automated user defined border delineation software (Siemens Argus Analysis Software, Siemens, Erlangen, Germany). The values obtained and their standard deviations were then recorded.

\section{CMR protocols}

The CMR protocols at centres 1 and 2 were pre-defined in the CYCLE-HD and CUDDLE studies respectively. Differences in scan parameters are due to local expertise in gaining the highest quality images possible with the fewest number of artefacts.

\section{CMR protocol centre 1}

All patients were imaged on a $3 \mathrm{~T}$ CMR platform (Skyra, Siemens Medical Imaging, Erlangen, Germany) using an 18-channel phased-array anterior coil. Patients were scanned on non-dialysis days, but not after the long-break. The CMR protocols for acquiring cine imaging and native T1 maps were as previously described [18], conforming to internationally recognized standards [22]. Electrographic gated breath-hold steady-state free procession long-axis cine images in 2, 3 and 4 chamber views were acquired. Short axis cine images covering the entire left ventricle were taken at $8 \mathrm{~mm}$ slice thickness, $2 \mathrm{~mm}$ gap, field of view $300 \times 400 \mathrm{~mm}$, matrix $208 \times 256$, repetition time $2.9 \mathrm{~ms}$, echo time $1.2 \mathrm{~ms}$, flip angle $64-79^{\circ}$, temporal resolution $<50 \mathrm{~ms}$, with 30 phases per cardiac cycle, in-plane image resolution $1.1 \times 1.5 \mathrm{~mm}$ to $1.3 \times 1.7 \mathrm{~mm}$.

T1 imaging parameters included acquisition of basal and mid T1 maps in 2 LV short-axis slices using the modified look-locker inversion recovery (MOLLI) sequence. Images were acquired using free-breathing with motion correction (MOCO), ECG-gated single-shot MOLLI sequence [23], with 3(3)3(3)5 sampling pattern and the following typical parameters: slice thickness $8.0 \mathrm{~mm}$, field of view $300 \times$ $400 \mathrm{~mm}$, flip angle $50^{\circ}$, minimum TI $120 \mathrm{~ms}$, inversiontime increment $80 \mathrm{~ms}$. MOLLI maps of the left ventricle were acquired at basal and mid-short-axis. The MOLLI sequence was chosen due to the technique's excellent inter and intra-observer variability at $3 \mathrm{~T}$ [24] and because of local expertise [14]. To minimize artefacts, acquisition was performed with the region of interest at isocentre, a small shim volume was applied around the myocardium, a large field of view $(400 \mathrm{~mm}$ ) was used, and imaging was repeated after changing the phase-encoding direction or resonance offset frequency if artefacts persisted.

\section{CMR protocol centre 2}

All participants were scanned on a $3 \mathrm{~T}$ CMR platform (Siemens Magnetom PRISMA, Siemens Medical Imaging, Erlangen, Germany) using a 16 channel phased-array anterior coil. As at centre 1 CMR imaging was performed on a non-dialysis day. Image acquisition was ECG-gated and as previously described [17]. T1 imaging parameters included acquisition of basal and mid T1 maps in 2 LV short-axis slices using a MOLLI sequence. Typical acquisition parameters were: slice thickness $6.0 \mathrm{~mm}$, field of view $360 \mathrm{x}$ $307 \mathrm{~mm}$, flip angle $35^{\circ}$, minimum T1 $180 \mathrm{~ms}$, inversiontime increment $80 \mathrm{~ms}$, repetition time $267.84 \mathrm{~ms}$, bandwidth 1085 Hertz/pixel.

\section{CMR scan analysis centre 1}

All scans were analysed offline by a single blinded observer using the software package $\mathrm{CMR}^{42}$ (Circle Cardiovascular Imaging, Calgary, Alberta, Canada). Image quality was assessed as being excellent, good, acceptable or poor. LV volumes and mass were quantified as previously described with epicardial and endocardial short axis cines at end- diastole and end-systole [25]. The native T1 parametric map derived from MOCO MOLLI images was used to assess native T1 signal due to superior intra and inter-observer variability as described by our group compared to analysing the $\mathrm{MOCO}$ series [14]. Using the CMR42 T1 characterization module, endocardial and epicardial borders were drawn on basal and mid-ventricular T1 parametric maps for each patient, with care taken to allow adequate margins of separation from tissue interfaces such as between the blood pool or epicardial fat and myocardium. The anterior right ventricular insertion point was then defined to automatically divide the basalventricular and mid-ventricular slices into 6 segments each according to the American Heart Association 16-segment model (Fig. 1). Each individual segment was assessed for the presence or absence of susceptibility and motion artefacts. After removal of any segments affected by artefact, an average $\mathrm{T} 1$ time for the whole of the myocardium was calculated from the mean of remaining segments.

\section{CMR scan analysis centre 2}

All scans were analysed offline by a single blinded observer using Siemens Argus Analysis software (Siemens, Erlangen, Germany). The image analysis technique was as previously described [17]. LV contours were defined on raw T1 images (the first image, with the lowest inversion time, allowing for optimal tissue blood-pool contrast) and copied into the parametric T1 maps [26, 27]. Using the anterior right ventricular insertion point as a reference, basal and mid T1 maps were segmented according to the American Heart Association 16-segment model and regions of interest were delineated by user-defined border delineation. The regions of interest were standardized to be of similar size and shape. T1 times were measured in each of the 6 basal and 6 mid segments as well as the blood pool, with care taken to delineate regions of interest with adequate margins of separation from tissue interfaces such as between the blood pool or epicardial fat and myocardium (Fig. 1). Each individual segment was assessed for the presence or absence of 


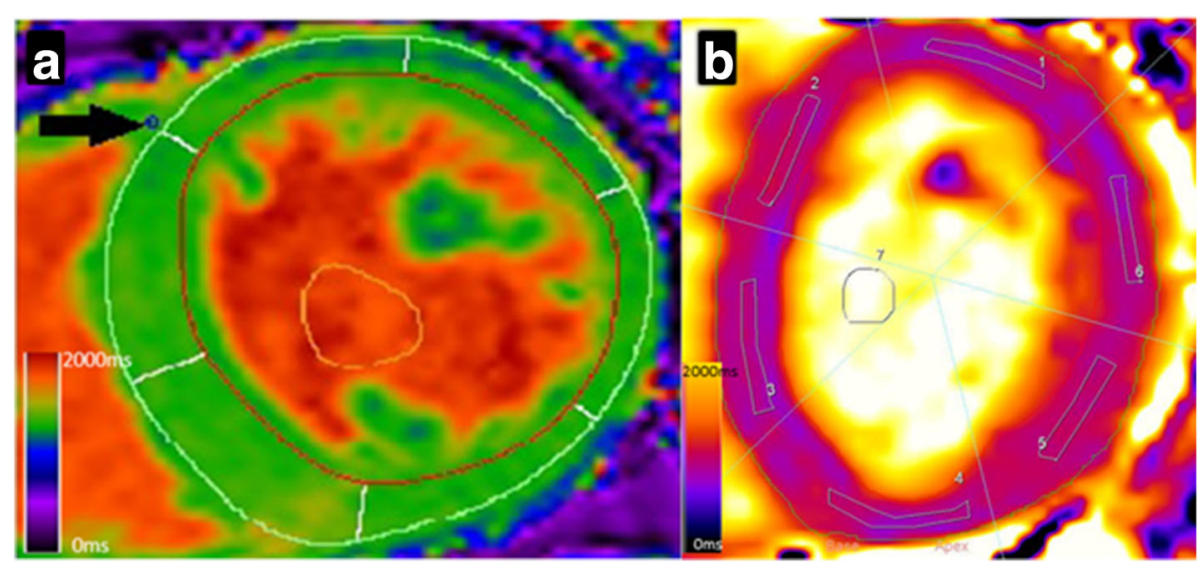

Fig. 1 a: Native T1 map analysis of a mid-ventricular slice with software package $\mathrm{CMR}^{42}$ at centre 1. Endocardial (red line) and epicardial (green line) contouring on native T1 parametric map. Segments (1-6) are calculated from defining the RV insertion point (arrow). b: Typical segmentation of a basal native T1 map site from centre 2.6 discrete regions of interest drawn within the myocardium for each segment

susceptibility and motion artefacts. After removal of any segments affected by artefact, an average T1 time for the whole of the myocardium was calculated from the mean of remaining segments.

\section{Statistical analysis}

Statistical analysis were undertaken using SPSS-22 software (Statistical Package for the Social Sciences, Chicago, IL, USA) and Graphpad Prism version 6.04 (GraphPad Software, Inc., La Jolla, CA, USA). Normality was assessed using the Shapiro-Wilk test, histograms, and Q-Q plots. Normally distributed data are expressed as mean \pm standard deviation and non-normally distributed data are expressed as median (interquartile range). Interstudy and inter-observer variability for subjects undergoing test-retest scans were compared using paired t-tests, co-efficients of variation and the BlandAltmann method [28]. Inter-centre differences between subject demographics and native T1 values were assessed with independent sample t-tests or Mann-Whitney $U$ tests for normally and non-normally distributed data respectively. Chi-squared tests and Fishers exact tests were used to assess for differences between nominal variables and are expressed as 'count' (\%). Correlations between variables were assessed using Pearson's and Spearman's-rank analysis for normally and non-normally distributed data respectively.

\section{Sample size justification}

A paired $t$-test sample size calculation was undertaken for the native $\mathrm{T} 1$ test-rest study, using the native $\mathrm{T} 1$ values from a previous study by our group conducted on the same $3 \mathrm{~T}$ MRI scanner (Skyra, Siemens Medical Imaging, Erlangen, Germany) [14]. To detect a $2.5 \%$ difference in native T1 times between test-retest scans $(27 \mathrm{~ms})$ with $90 \%$ power requires 8 patients to undergo test-retest scans. Ten patients were therefore recruited to undergo test-retest scans.

\section{Results}

The demographic data for all patients from both centres are shown in Table 1 . There were no statistically significant differences between the demographics of the groups. There was a trend towards longer dialysis vintage in participants

Table 1 Demographic details from both study centres

\begin{tabular}{lll}
\hline Variable & Centre $1(n=10)$ & Centre $2(n=10)$ \\
\hline Age (Years) & $57.8 \pm 15$ & $58 \pm 13.5$ \\
Male (\%) & $8(80 \%)$ & $7(70 \%)$ \\
HR (bpm) & $76 \pm 14$ & $68 \pm 11.6$ \\
SBP (mmHg) & $143 \pm 33$ & $143 \pm 19$ \\
DBP (mmHg) & $80 \pm 15$ & $72 \pm 13$ \\
Dialysis Vintage (Months) & $26 \pm 26.2$ & $9 \pm 3.9$ \\
Past Medical and Drug History & & \\
HTN (n,\%) & $8(80 \%)$ & $6(60 \%)$ \\
Diabetes (n,\%) & $3(30 \%)$ & $2(20 \%)$ \\
Previous Ml (n,\%) & $1(10 \%)$ & $2(20 \%)$ \\
CAD (n,\%) & $3(30 \%)$ & $3(30 \%)$ \\
PVD (n,\%) & $0(0 \%)$ & $1(10 \%)$ \\
ACEi (n,\%) & $3(30 \%)$ & $1(10 \%)$ \\
ARB (n\%) & $1(10 \%)$ & $0(0 \%)$ \\
Diuretic (n,\%) & $1(10 \%)$ & $2(20 \%)$ \\
Beta Blocker (n,\%)* & $4(40 \%)$ & $7(70 \%)$ \\
Statin (n,\%) & $4(40 \%)$ & $5(50 \%)$ \\
Calcium Channel Blocker (n,\%) & $3(30 \%)$ & $2(20 \%)$ \\
Number of antihypertensives & $0.96 \pm 0.9$ & $1.1 \pm 0.9$ \\
\hline Mean vas wth stand & & \\
\hline
\end{tabular}

Mean values with standard deviation expressed as $n \pm S D$. N, \% = Chi-squared $+\%$. No significant differences were observed between any baseline demographic details

bpm beats per minute, $A C E i$ angiotensin converting enzyme inhibitor, $A R B$ angiotensin receptor blocker, $C A D$ coronary artery disease, $D B P$ diastolic blood pressure, $H R$ heart rate, HTN hypertension, $M I$ myocardial infarction, $P V D$ peripheral vascular disease, SBP systolic blood pressure

${ }^{*}$ statistically significant difference between groups $(P<0.05)$ 
from centre 1 compared to those in centre 2, but these difference did not reach statistical significance. There were no differences between the basal or the mid-ventricular native T1 times for HD patients between centre 1 and centre 2 respectively (basal T1 $1280.5 \mathrm{~ms} \pm 36.6 \mathrm{vs} 1276.3 \mathrm{~ms} \pm 32.3$, $P=0.62$ and mid T1 $1282.6 \mathrm{~ms} \pm 38.1$ vs $1283.4 \pm 39.3, P=$ 0.42 ). All scans were analysable and included in the analysis.

\section{Inter-study reproducibility of LV masses and volumes}

The mean interval between scans for patients undergoing test-retest inter-study reproducibility at centre 1 was $7 \pm$ 4 days. Image quality was either good $(n=4)$ or excellent $(n$ $=16$ ) and of sufficient quality for quantitative analysis. The inter-study reproducibility, including coefficients of variation (CoV), bias and limits of agreement for LV mass, LVEDV, LVESV and LVEF are shown in Table 2. Bland-Altman plots did not show evidence of systematic bias, with all data points within 95\% confidence intervals (Fig. 2). Interstudy mean difference and 95\% confidence intervals of LVEDV and weight were $11.79 \mathrm{ml}(5.6,18.0)$ and $0.5 \mathrm{~kg}(0.1,0.8)$ respectively. There was a significant correlation between change in LVEDV $(\triangle \mathrm{LVEDV})$ and change in weight ( $\Delta$ weight) between scans $(r$ $=0.682, P=0.03)$, but no association was found between change in LV mass ( $\triangle$ LVMass) and $\triangle \mathrm{LVEDV}$ or $\Delta$ weight $(r=$ $0.16, P=0.67$ and $r=0.12, P=0.73$ respectively) (Fig. 3).

\section{Inter-study reproducibility, inter-observer and} intra-observer variability of mid-ventricular native $\mathrm{T} 1$

Six out of 120 native T1 segments from mid-ventricular native $\mathrm{T} 1$ parametric maps were excluded from analysis due to artefact. The same segments were excluded from analysis by the two readers in the inter-observer analysis (one segment from interventricular septum and five from the lateral wall). The inter-study reproducibility, inter-observer and intraobserver variability of mid-ventricular native $\mathrm{T} 1$ values, including $\mathrm{CoV}$, bias and limits of agreement for mid-LV native T1 values are shown in Table 2. Bland-Altman plots did not show evidence of any systematic bias, with all data points within 95\% confidence intervals (Fig. 2). There was no significant correlation between change in mid-ventricular native T1 $(\Delta \mathrm{T} 1)$ or change in LVEDV $(\triangle \mathrm{LVEDV})$ and change in weight ( $\Delta$ weight) between scans $(r=-0.14, P=0.7$ and $r=0.2, P=0.6$, respectively) (Fig. 4). Linear regression confirmed $\triangle \mathrm{T} 1$ was unaffected by both $\triangle \mathrm{LVEDV}$ and $\Delta$ weight (adj $R 2=0.1, P=0.71$ and adj $R 2=0.08, P=0.59$ ). Based on these results, to detect a $2.5 \%$ difference in native $\mathrm{T} 1$ values with $90 \%$ power would require 13 patients and to detect a $5 \%$ difference in native $\mathrm{T} 1$ values with $90 \%$ power would require 5 patients (alpha error $=0.05$ for both).

\section{Inter-centre measurement variability of basal and mid-ventricular native T1}

Twenty-five out of 240 native T1 segments were excluded from analysis due to artefact $(9$ segments were excluded from the interventricular septum and 16 from the later wall). The two readers agreed on the analysability of $220 / 240$ segments reviewed (91.7\%). The inter-centre variability of native $\mathrm{T} 1$ analysis techniques of basal and mid-ventricular native T1 values, including $\mathrm{CoV}$, bias and limits of agreement are shown in Table 3. Bland-Altman plots did not show evidence of systematic bias (Additional file 1: Appendix 1), although single data points fell just outside the $95 \%$ confidence intervals for mid-ventricular native T1 of centre 1 and for basalventricular and mid-ventricular native T1 for centre 2 .

\section{$\mathrm{T} 1$ values of standard phantom between centres}

Excellent agreement was seen for the phantom results obtained at both centres with no clinically relevant differences between scanners. Allowing for standard deviations, the phantom considered representative of a typical myocardial tissue $\mathrm{T} 1$ time, gave very similar $\mathrm{T} 1$ values at both sites (centre 1: $1192.7 \pm 7.5 \mathrm{~ms}$, centre 2: $1205.5 \pm$ $5 \mathrm{~ms}$ ). For the phantom blood pool T1 times obtained

Table 2 Interstudy reproducibility of LV mass, volumes and mid-ventricular native T1 values and Inter-observer and intra-observer variability of mid-ventricular native T1 values

\begin{tabular}{|c|c|c|c|c|c|}
\hline Parameter & Study 1 & Study 2 & $\mathrm{CoV}$ & BIAS \pm SD Difference & BA Limits of Agreement \\
\hline \multicolumn{6}{|l|}{ Inter-study reproducibility } \\
\hline LV Mass (diastolic) (g) & $95.2 \pm 22.0$ & $95.5 \pm 22.7$ & $1.0 \%$ & $-0.3 \pm 1.96$ & $-4.5-3.5$ \\
\hline LVEDV (ml) & $139.3 \pm 21.0$ & $138.5 \pm 27.8$ & $5.2 \%$ & $0.8 \pm 15.2$ & $-28.9-30.5$ \\
\hline LVESV (ml) & $64.3 \pm 16.3$ & $64.2 \pm 19.7$ & $5.6 \%$ & $0.1 \pm 7.6$ & $-14.8-15$ \\
\hline LVEF (\%) & $54.3 \pm 7.2$ & $54.4 \pm 6.8$ & $1.1 \%$ & $-0.1 \pm 1.3$ & $-2.6-2.4$ \\
\hline Mid-ventricular native T1 (ms) & $1267.8 \pm 35.4$ & $1270.7 \pm 30.5$ & $0.7 \%$ & $-2.9 \pm 17.5$ & $-37.2-31.3$ \\
\hline \multicolumn{6}{|l|}{ Inter-observer variability } \\
\hline Mid-ventricular native T1 (ms) & $1267.6 \pm 35.4$ & $1271 \pm 34.8$ & $0.3 \%$ & $-3.4 \pm 6.2$ & $-15.6-8.8$ \\
\hline \multicolumn{6}{|l|}{ Intra-observer variability } \\
\hline Mid-ventricular native $\mathrm{T1}$ (ms) & $1267 \pm 34.3$ & $1266 \pm 35.5$ & $0.4 \%$ & $0.66 \pm 11.7$ & $-22.3-23.7$ \\
\hline
\end{tabular}

Mean values with standard deviation expressed as $\mathrm{n} \pm \mathrm{SD}$

$B A$ Bland-Altman, LV left ventricular, LVEDV left ventricular end-diastolic volume, LVESV left ventricular end-systolic volume, LVEF left ventricular ejection fraction 

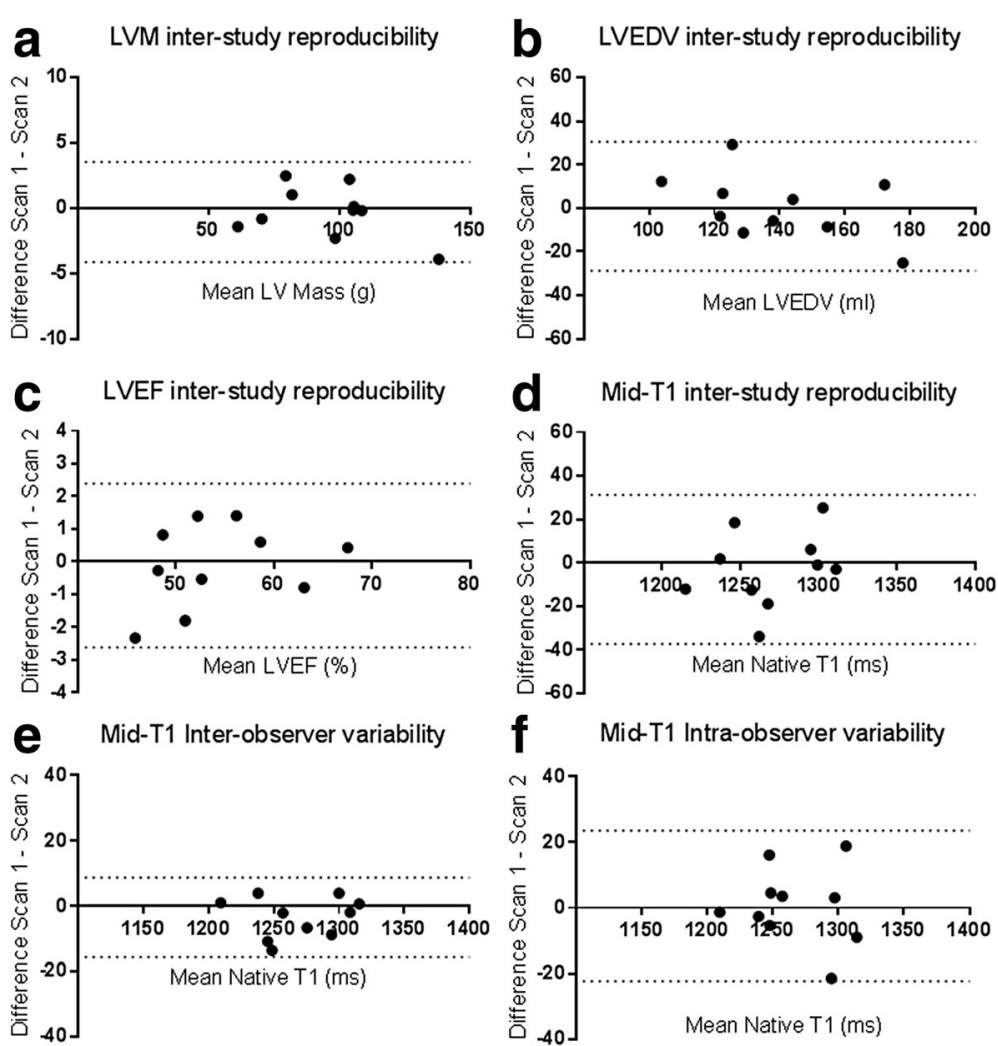

Fig. 2 Bland-Altman plots for: a: Inter-study reproducibility left ventricular mass, b: Inter-study reproducibility left ventricular end-diastolic volume, c: Inter-study reproducibility left ventricular ejection fraction, $\mathbf{d}$ : Inter-study reproducibility mid-ventricular native T1, e: inter-observer variability of mid-ventricular native $\mathrm{T} 1$ and $\mathbf{f}$ : intra-observer variability of mid-ventricular native T1

were again equivalent at each centre: (centre 1: $1899.2 \pm$ $6.9 \mathrm{~ms}$, centre 2: $1907.9 \pm 10.7 \mathrm{~ms})$.

\section{Discussion}

In this study we have shown, for the first time in patients with ESRD on $\mathrm{HD}$, that the reproducibility of native T1 values are excellent. The reproducibility values for $\mathrm{T} 1$ mapping using the MOLLI sequence compare favourably with those previously reported. Previous CoVs for interstudy reproducibility have been between 1.5 and $8.4 \%$, [14-16, 29] compared with $0.7 \%$ in this report. As previously described, native $\mathrm{T} 1$ is related to water content of the relevant tissue [30] and the finding in this study that $\Delta \mathrm{T} 1$ between test-re-test scans was unrelated to changes in fluid status suggests that changes in fluid status at the levels we have seen do not directly affect native $\mathrm{T} 1$ values in HD patients, although we accept that this study is only in 10 patients. Whilst the absolute changes in inter-study fluid status that we report are not large, they are the types of fluctuations in fluid you would expect to see in HD patients scanned on a non-dialysis day on week apart and therefore clinically relevant. This is a crucial observation in defining the reliability of $\mathrm{T} 1$ time as a potential measure of myocardial fibrosis in this patient group.
We have demonstrated that standardization of native $\mathrm{T} 1$ mapping analysis between centres is eminently possible. The agreement between the analysis techniques of centres is encouraging. Both techniques define segmental native T1 values for basal- and mid-ventricular slices as per the American Heart Association model (Fig. 1), with the average of these segments taken to be the mean native T1 for each slice. When areas of artefact are identified, affected segments can be excluded with the mean native T1 calculated from remaining unaffected segments. This way of assessing native $\mathrm{T} 1$ is likely to be more reproducible than alternative methods, where single regions of interest are drawn in the inter-ventricular septum [31]. Such methods are prone to sampling differences in native $\mathrm{T} 1$ times depending on where the region of interest is drawn. We have previously demonstrated that septal native $\mathrm{T} 1$ is significantly higher than non-septal native $\mathrm{T} 1$ in $\mathrm{HD}$ patients [18]. Moreover, a recent paper by Rauhalammi et al showed that native $\mathrm{T} 1$ values are significantly higher in the interventricular septum of health volunteer patients than nonseptal myocardium [27] so analysis techniques that measure only the interventricular septal native $\mathrm{T} 1$ cannot be thought of as equivalent to mean circumferential ventricular native $\mathrm{T} 1$ values. The phantom analysis conducted at centre 1 and 

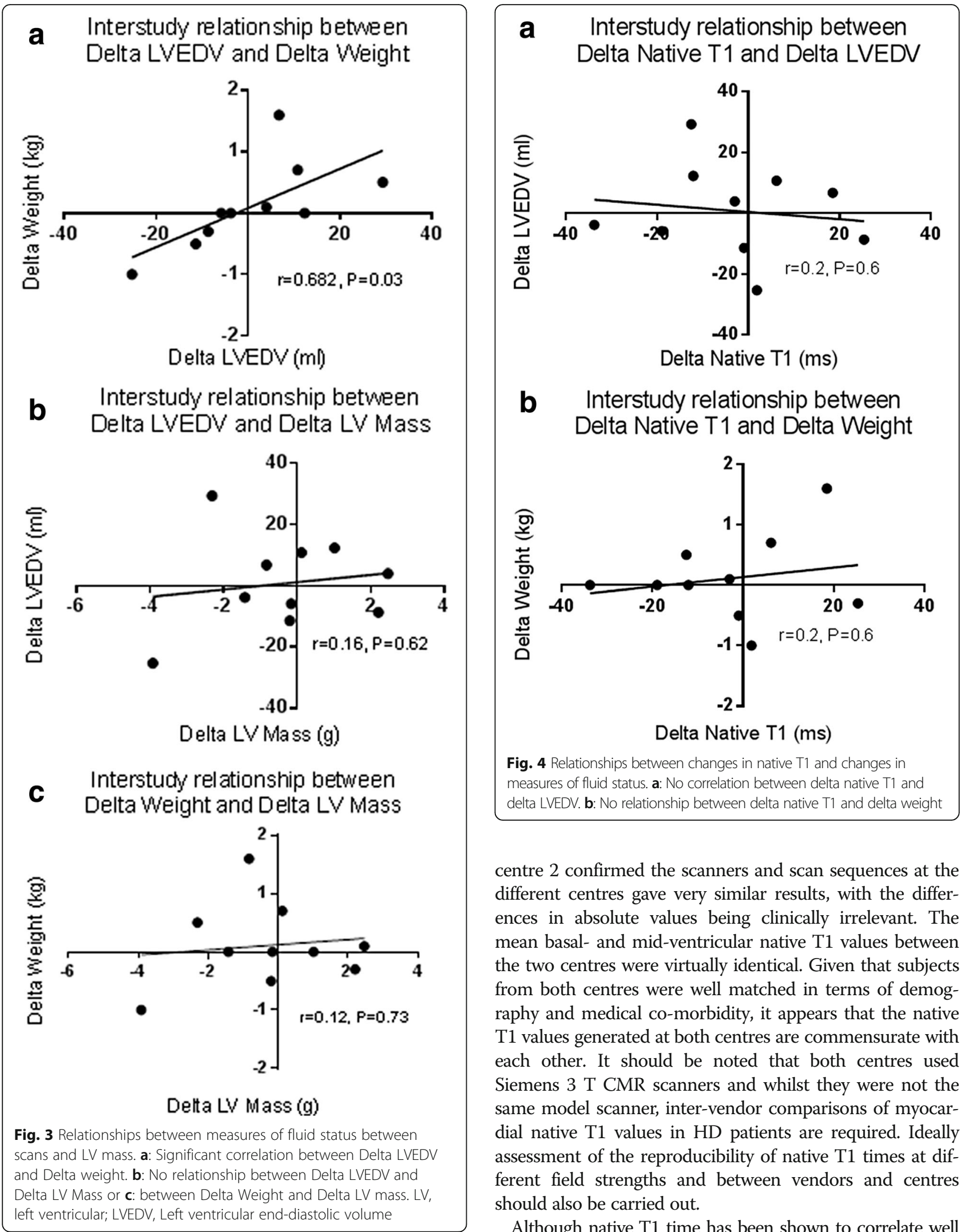

centre 2 confirmed the scanners and scan sequences at the different centres gave very similar results, with the differences in absolute values being clinically irrelevant. The mean basal- and mid-ventricular native $\mathrm{T} 1$ values between the two centres were virtually identical. Given that subjects from both centres were well matched in terms of demography and medical co-morbidity, it appears that the native $\mathrm{T} 1$ values generated at both centres are commensurate with each other. It should be noted that both centres used Siemens $3 \mathrm{~T}$ CMR scanners and whilst they were not the same model scanner, inter-vendor comparisons of myocardial native $\mathrm{T} 1$ values in $\mathrm{HD}$ patients are required. Ideally assessment of the reproducibility of native $\mathrm{T} 1$ times at different field strengths and between vendors and centres should also be carried out.

Although native T1 time has been shown to correlate well with histological levels of myocardial fibrosis in diseases of pressure overload [12] we still do not have histological 
Table 3 Inter-centre variability of basal and mid-ventricular native T1 analysis techniques

\begin{tabular}{|c|c|c|c|c|c|}
\hline Parameter & Centre 1 analysis & Centre 2 analysis & $\mathrm{CoV}$ & Bias \pm SD Difference & BA Limits of Agreement \\
\hline \multicolumn{6}{|c|}{ Centre 1 native $\mathrm{T} 1$ images } \\
\hline Basal native T1 (ms) & $1283.8 \pm 38.7$ & $1281.4 \pm 39.6$ & $0.8 \%$ & $2.4 \pm 21.5$ & $-39.7-44.6$ \\
\hline Mid native T1 (ms) & $1281.3 \pm 39.6$ & $1279.8 \pm 35.6$ & $1.2 \%$ & $1.5 \pm 31.2$ & $-59.7-62.6$ \\
\hline \multicolumn{6}{|c|}{ Centre 2 native $\mathrm{T} 1$ images } \\
\hline Basal native $\mathrm{T} 1$ (ms) & $1290.4 \pm 36.7$ & $1276.4 \pm 42.5$ & $0.8 \%$ & $14.1 \pm 17.2$ & $-19.7-47.8$ \\
\hline Mid native T1 (ms) & $1281.3 \pm 31.6$ & $1271.3 \pm 33.9$ & $0.9 \%$ & $10 \pm 23.1$ & $-35-55.2$ \\
\hline
\end{tabular}

Mean values with standard deviation expressed as $\mathrm{n} \pm \mathrm{SD}$

BA Bland-Altman

confirmation that this is the case for HD patients; indeed low grade inflammation may increase native T1 [32]. Low grade inflammation is known to be a feature of CKD and for patients with ESRD on HD [33], so histological studies are required to confirm increased T1 times are due to myocardial fibrosis and not low grade inflammation or an additional disease process such as amyloidosis [34]. Whilst our results suggest inter-compartmental fluid shifts do not affect native T1 values, further, confirmatory studies should be considered, including studies that look at the acute effects of $\mathrm{HD}$ and ultrafiltration on native $\mathrm{T} 1$ values.

The inter-study reproducibility of traditional CMR measures of LV structure and function, including LV mass, LVEDV and LVEF were also excellent as has been shown previously for patients with ESRD [35]. The reproducibility of LV mass is particularly important as LV mass is known to be overestimated when imaged with echocardiography [36]. These findings confirm previous studies in HD patients showing conservation of measurement of LV mass with CMR in patients with differing LVEDV's and fluid and loading status [35].

In 2011, Sado et al outlined a framework for development of imaging techniques to measure myocardial fibrosis, to ensure the robustness of the techniques [37]. They suggested that to be considered a reliable imaging biomarker of myocardial fibrosis, techniques should: detect changes in established disease states compared with controls; correlate with cardiac markers fibrosis (e.g., diastolic function, LVH); correlate with blood biomarkers of cardiac fibrosis; be able to track changes over time; be standardized in the way they are carried out (inter-vendor/inter-centre); be proven to compare closely with histological specimens from human subjects; and changes in the imaging biomarker should track changes in the disease after treatment. Work by our respective groups has already shown that native $\mathrm{T} 1$ values are higher than healthy and co-morbidity matched controls $[17,18]$. Rutherford et al demonstrated that global native $\mathrm{T} 1$ values in HD correlate with LV mass indices $(r=0.452$, $P=0.008$ ), and that septal T1 values correlate with predialysis high-sensitive troponin $\mathrm{T}(r=0.397, P=0.027)$ [17]. In addition to this, Graham-Brown et al showed that native $\mathrm{T} 1$ values correlate with measures of global circumferential and longitudinal strain $(r=0.41, P=0.002, r=0.55, P<$ $0.001)$ and septal native T1 correlated with septal systolic strain $(r=0.46, P<0.001)[18]$.

Following the framework outlined by Sado et al, histological confirmation of the relationship between myocardial fibrosis and native T1 mapping now appears to be the final step required before longitudinal and interventional studies can be undertaken to define whether native T1 mapping is able to: track progression of myocardial fibrosis over time; assess how baseline or changes in native $\mathrm{T} 1$ values relate to outcomes; whether interventions that attempt to modify/improve fibrosis are measurable by native $\mathrm{T} 1$ mapping; and whether this modification improves outcomes for patients. The excellent reproducibility of native T1 mapping makes it a biomarker with great potential for the measurement of myocardial fibrosis in HD patients. Based on the results we have presented, only small numbers of patients would be required to adequately power interventional clinical trials that seek to reduce myocardial fibrosis. Whilst historically LV mass has been the surrogate end-point for clinical trial work that sought to improve $\mathrm{CV}$ outcomes, a recent systematic review and meta-analysis has called into question whether interventions that improve LV mass actually improve outcomes for patients with CKD and ESRD [5]. It should be noted that the neutral result of this meta-analysis might be due to inadequate power. Post-mortem histo-pathological studies have shown us that extent of myocardial fibrosis is the best predictor of outcome in patients with CKD and ESRD [6] and there is evidence in animal models, humans and even HD patients that regression of LVH is accompanied by reductions in measures of myocardial fibrosis [3841]. Given that multiple studies have suggested that reducing LV mass improves outcomes for patients with ESRD [42], it could be that previous trials that have shown reductions in LV mass have actually just reflected a crude measure of a reduction in myocardial fibrosis content and this has led to the conflicting results about the importance of LV mass reduction and $\mathrm{CV}$ risk that has recently been reported. Given the central association between fibrosis, LVH, morbidity and mortality in HD patients, being able to reliably measure diffuse myocardial fibrosis is crucial to understanding its potential as a research and clinical end point. 


\section{Conclusion}

Native T1 mapping is an extremely reproducible technique in HD patients. Native T1 times seem to be unaffected by the changes in fluid status to which HD patients are prone. Further work is required to determine whether native myocardial T1 is related to prognosis in ESRD and whether interventions that reduce $\mathrm{T} 1$ are associated with improved outcomes.

\section{Additional file}

Additional file 1: Appendix 1. Bland-Altman plots for variability of intercentre analysis techniques of A: Basal-ventricular native $\mathrm{T} 1$ values of centre 1. B: Mid-ventricular native T1 values of centre 1. C: Basal-ventricular native T1 values of centre 2. D: Mid-ventricular native T1 values of centre 2. (TIF 574 kb)

\section{Acknowledgements}

Not applicable

\section{Funding}

This study is independent research arising from a Clinician Scientist Award (Dr James Burton, CS-2013-13-014) supported by the NIHR, and a grant from Kidney Research UK (Research Innovation Grant IN02/2013). This work is also supported by the NIHR Leicester Cardiovascular Biomedical Research Unit based at University Hospitals of Leicester and the University of Leicester and the NIHR Diet, Lifestyle \& Physical Activity Biomedical Research Unit based at University Hospitals of Leicester and Loughborough University.

The views expressed in this publication are those of the authors and not necessarily those of the National Health Service, the National Institute for Health Research or the Department of Health.

\section{Availability of data and materials}

The datasets generated and/or analysed during the current study are not publicly available but are available from the corresponding author on reasonable request.

\section{Authors' contributions \\ MGB - Manuscript preparation, data collection and analysis, study design, final article approval. ER - Manuscript review, data collection and analysis, final article approval. EL - Data collection and analysis, final article approval. DM - Data collection, final article approval. DC - Data collection, final article approval. DS - Manuscript review, final article approval. CM - MRI sequence comparison and review, data collection, final article approval. KM - MRI sequence comparison and review, final article approval. SC - MRI sequence comparison and review, final article approval. CB - Study design, manuscript review, final manuscript approval. JM - Native T1 phantom design and organisation, final article approval. PB - Study design, data analysis, manuscript review, final manuscript approval. JB - Study design, data analysis, manuscript review, final manuscript approval. GM - Study design, data analysis, manuscript review, final manuscript approval.}

\section{Competing interests}

The authors declare that they have no competing interests.

\section{Consent for publication}

Not Applicable.

\section{Ethics approval and consent to participate}

Patients from centre 1 were recruited as a part of the CYCYLE-HD study (ISRCTN 11299707) [21]. The study was given ethical approval by the NHS Research Ethics Committee East Midlands (Northampton; REC ref: 14/EM/1190). Patients from centre 2 were recruited as part of the observational cardiac uraemic fibrosis detection in dialysis patients study (CUDDLE study ISCRTN99591655). The study was approved by the West of Scotland Ethics committee (WoS 13/ WS/0301). All participants gave written and informed consent.

\section{Author details}

1John Walls Renal Unit, University Hospitals Leicester NHS Trust, Leicester, UK. ${ }^{2}$ Department of Infection Immunity and Inflammation, School of Medicine and Biological Sciences, University of Leicester, Leicester LE1 9HN, UK. ${ }^{3}$ National Centre for Sport and Exercise Medicine, School of Sport, Exercise and Health Sciences, Loughborough University, Loughborough, UK. ${ }^{4}$ BHF Cardiovascular Research Centre, Institute of Cardiovascular and Medical Sciences, University of Glasgow, 126 University Place, Glasgow, UK. ${ }^{5}$ The Glasgow Renal \& Transplant Unit, Queen Elizabeth University Hospital, 1345 Govan Road, Glasgow, UK. 'Department of Cardiovascular Sciences, University of Leicester and NIHR Leicester Cardiovascular Biomedical Research Unit, Glenfield Hospital Leicester, Leicester, UK. ${ }^{7}$ Clinical Physics, NHS Greater Glasgow and Clyde, Glasgow, UK. ${ }^{8}$ West of Scotland Heart and Lung Centre, Golden Jubilee National Hospital, Clydebank, UK. ${ }^{9}$ UCL Institute of Cardiovascular Science, University College London, London, UK.

Received: 27 January 2017 Accepted: 2 February 2017

Published online: 27 February 2017

\section{References}

1. U.S. Renal Data System. USRDS 2006 Annual Data Report: Atlas of End-Stage Renal Disease in the United States. 2006.

2. Glassock RJ, Pecoits-Filho R, Barberato SH. Left ventricular mass in chronic kidney disease and ESRD. Clin J Am Soc Nephrol. 2009;4 Suppl 1:S79-91.

3. Pierdomenico SD, Cuccurullo F. Risk reduction after regression of echocardiographic left ventricular hypertrophy in hypertension: a metaanalysis. Am J Hypertens. 2010;23(8):876-81.

4. Mancini GB, Dahlof B, Diez J. Surrogate markers for cardiovascular disease: structural markers. Circulation. 2004;109(25 Suppl 1):IV22-30.

5. Badve SV, Palmer SC, Strippoli GF, Roberts MA, Teixeira-Pinto A, Boudville N, et al. The Validity of Left Ventricular Mass as a Surrogate End Point for AllCause and Cardiovascular Mortality Outcomes in People With CKD: A Systematic Review and Meta-analysis. Am J Kidney Dis. 2016;68(4):554-63.

6. Mall G, Huther W, Schneider J, Lundin P, Ritz E. Diffuse intermyocardiocytic fibrosis in uraemic patients. Nephrol Dial Transplant. 1990;5(1):39-44.

7. Aoki J, Ikari Y, Nakajima H, Mori M, Sugimoto T, Hatori M, et al. Clinical and pathologic characteristics of dilated cardiomyopathy in hemodialysis patients. Kidney Int. 2005;67(1):333-40.

8. Mark P, Johnston N, Groenning B, Foster J, Blyth K, Martin T, et al. Redefinition of uremic cardiomyopathy by contrast-enhanced cardiac magnetic resonance imaging. Kidney Int. 2006:69(10):1839-45.

9. Kribben A, Witzke O, Hillen U, Barkhausen J, Daul AE, Erbel R. Nephrogenic systemic fibrosis: pathogenesis, diagnosis, and therapy. J Am Coll Cardiol. 2009;53(18):1621-8.

10. Kehr E, Sono M, Chugh SS, Jerosch-Herold M. Gadolinium-enhanced magnetic resonance imaging for detection and quantification of fibrosis in human myocardium in vitro. Int J Cardiovasc Imaging. 2008;24(1):61-8.

11. Flett AS, Sado DM, Quarta G, Mirabel M, Pellerin D, Herrey AS, et al. Diffuse myocardial fibrosis in severe aortic stenosis: an equilibrium contrast cardiovascular magnetic resonance study. Eur Heart J Cardiovasc Imaging. 2012;13(10):819-26.

12. Bull S, White SK, Piechnik SK, Flett AS, Ferreira VM, Loudon M, et al. Human non-contrast $\mathrm{T} 1$ values and correlation with histology in diffuse fibrosis. Heart. 2013:99(13):932-7.

13. Hinojar R, Varma N, Child N, Goodman B, Jabbour A, Yu CY, et al. T1 Mapping in Discrimination of Hypertrophic Phenotypes: Hypertensive Heart Disease and Hypertrophic Cardiomyopathy: Findings From the International T1 Multicenter Cardiovascular Magnetic Resonance Study. Circ Cardiovasc Imaging. 2015 8(12):10.1161/CIRCIMAGING.115.003285.

14. Singh A, Horsfield MA, Bekele S, Khan JN, Greiser A, McCann GP. Myocardial $\mathrm{T} 1$ and extracellular volume fraction measurement in asymptomatic patients with aortic stenosis: reproducibility and comparison with age-matched controls. Eur Heart J Cardiovasc Imaging. 2015;16(7):763-70.

15. Rogers T, Dabir D, Mahmoud I, Voigt T, Schaeffter T, Nagel E, et al. Standardization of T1 measurements with MOLLI in differentiation between health and disease-the ConSept study. J Cardiovasc Magn Reson. 2013;15(1):1.

16. Pica S, Sado DM, Maestrini V, Fontana M, White SK, Treibel T, et al. Reproducibility of native myocardial T1 mapping in the assessment of Fabry disease and its role in early detection of cardiac involvement by cardiovascular magnetic resonance. J Cardiovasc Magn Reson. 2014;16:99-014-0099-4. 
17. Rutherford E, Talle MA, Mangion K, Bell E, Rauhalammi SM, Roditi G, et al. Defining myocardial tissue abnormalities in end-stage renal failure with cardiac magnetic resonance imaging using native T1 mapping. Kidney Int. 2016;90(4): 845-52.

18. Graham-Brown MP, March DS, Churchward DR, Stensel DJ, Singh A, Arnold R, et al. Novel cardiac nuclear magnetic resonance method for noninvasive assessment of myocardial fibrosis in hemodialysis patients. Kidney Int. 2016;90(4):835-44.

19. Shroff GR, Raggi P. Exploring the elusive link between subclinical fibrosis and clinical events in end-stage renal disease: does cardiac magnetic resonance imaging hold the key? Kidney Int. 2016;90(4):729-32.

20. Captur G, Gatehouse P, Kellman P, Heslinga FG, Keenan K, Bruehl R, et al. A $\mathrm{T} 1$ and ECV phantom for global T1 mapping quality assurance: The T 1 mapping and ECV standardisation in CMR (T1MES) program. J CardiovasC Magn Reson. 2016;18(1):1.

21. Graham-Brown M, March D, Churchward D, Young H, Dungey M, Lloyd S, et al. Design and methods of CYCLE-HD: improving cardiovascular health in patients with end stage renal disease using a structured programme of exercise: a randomised control trial. BMC Nephrol. 2016;17(1):1.

22. Kramer CM, Barkhausen J, Flamm SD, Kim RJ, Nagel E. Standardized cardiovascular magnetic resonance (CMR) protocols 2013 update. J Cardiovasc Magn Reson. 2013;15(1):1.

23. Messroghli DR, Radjenovic A, Kozerke S, Higgins DM, Sivananthan MU, Ridgway JP. Modified Look-Locker inversion recovery (MOLLI) for highresolution T1 mapping of the heart. Magn Reson Med. 2004;52(1):141-6.

24. Dabir D, Child N, Kalra A, Rogers T, Gebker R, Jabbour A, et al. Reference values for healthy human myocardium using a T1 mapping methodology: results from the International T1 Multicenter cardiovascular magnetic resonance study. J Cardiovasc Magn Reson. 2014;16(1):69.

25. Singh A, Steadman CD, Khan JN, Horsfield MA, Bekele S, Nazir SA, et al. Intertechnique agreement and interstudy reproducibility of strain and diastolic strain rate at 1.5 and 3 tesla: A comparison of feature-tracking and tagging in patients with aortic stenosis. J Magn Reson Imaging. 2015;41(4):1129-37.

26. Carrick D, Haig C, Rauhalammi S, Ahmed N, Mordi I, McEntegart M, et al. Prognostic significance of infarct core pathology revealed by quantitative non-contrast in comparison with contrast cardiac magnetic resonance imaging in reperfused STelevation myocardial infarction survivors. Eur Heart J. 2016:37(13):1044-59.

27. Rauhalammi SM, Mangion K, Barrientos PH, Carrick DJ, Clerfond G, McClure J, et al. Native myocardial longitudinal (T1) relaxation time: Regional, age, and sex associations in the healthy adult heart. J Magn Reson Imaging. 2016;44(3):5418.

28. Bland JM, Altman D. Statistical methods for assessing agreement between two methods of clinical measurement. Lancet. 1986;327(8476):307-10.

29. Liu S, Han J, Nacif MS, Jones J, Kawel N, Kellman P, et al. Diffuse myocardial fibrosis evaluation using cardiac magnetic resonance T1 mapping: sample size considerations for clinical trials. J Cardiovasc Magn Reson. 2012;14(1):1.

30. Piechnik SK, Ferreira VM, Dall'Armellina E, Cochlin LE, Greiser A, Neubauer S, et al. Shortened Modified Look-Locker Inversion recovery (ShMOLLI) for clinical myocardial T1-mapping at 1.5 and $3 \mathrm{~T}$ within a 9 heartbeat breathhold. J Cardiovasc Magn Reson. 2010;12(1):1.

31. Edwards NC, Moody WE, Yuan M, Hayer MK, Ferro CJ, Townend JN, et al. Diffuse Interstitial Fibrosis and Myocardial Dysfunction in Early Chronic Kidney Disease. Am J Cardiol. 2015;115(9):1311-7.

32. Hinojar R, Foote L, Ucar EA, Jackson T, Jabbour A, Yu C, et al. Native T1 in discrimination of acute and convalescent stages in patients with clinical diagnosis of myocarditis: a proposed diagnostic algorithm using CMR. J Am Coll Cardiol Img. 2015;8(1):37-46.

33. Caglar K, Hakim RM, Ikizler TA. Approaches to the reversal of malnutrition, inflammation, and atherosclerosis in end-stage renal disease. Nutr Rev. 2002; 60(11):378-87

34. Karamitsos TD, Piechnik SK, Banypersad SM, Fontana M, Ntusi NB, Ferreira VM, et al. Noncontrast T1 mapping for the diagnosis of cardiac amyloidosis. J Am Coll Cardiol Img. 2013;6(4):488-97.

35. Hunold P, Vogt FM, Heemann UW, Zimmermann U, Barkhausen J. Myocardial mass and volume measurement of hypertrophic left ventricles by MRI-study in dialysis patients examined before and after dialysis. J Cardiovasc Magn Reson. 2003;5(4):553-61.

36. Mclntyre CW, Odudu A, Eldehni MT. Cardiac assessment in chronic kidney disease. Curr Opin Nephrol Hypertens. 2009;18(6):501-6.

37. Sado DM, Flett AS, Moon JC. Novel imaging techniques for diffuse myocardial fibrosis. Futur Cardiol. 2011;7(5):643-50.
38. Linz W, Wiemer G, Schaper J, Zimmermann R, Nagasawa K, Gohlke P, et al. Angiotensin converting enzyme inhibitors, left ventricular hypertrophy and fibrosis. Mol Cell Biochem. 1995;147(1-2):89-97.

39. Simko F, Pechanova O. Remodelling of the heart and vessels in experimental hypertension: advances in protection. J Hypertens. 2010;28 Suppl 1:S1-6.

40. Krayenbuehl HP, Hess OM, Monrad ES, Schneider J, Mall G, Turina M. Left ventricular myocardial structure in aortic valve disease before, intermediate, and late after aortic valve replacement. Circulation. 1989;79(4):744-55.

41. Chan CT, Li GH, Valaperti A, Liu P. Intensive Hemodialysis Preserved Cardiac injury. ASAIO J. 2015;61(5):613-9.

42. London GM, Pannier B, Guerin AP, Blacher J, Marchais SJ, Darne B, et al. Alterations of left ventricular hypertrophy in and survival of patients receiving hemodialysis: follow-up of an interventional study. J Am Soc Nephrol. 2001;12(12):2759-67.

\section{Submit your next manuscript to BioMed Central and we will help you at every step:}

- We accept pre-submission inquiries

- Our selector tool helps you to find the most relevant journal

- We provide round the clock customer support

- Convenient online submission

- Thorough peer review

- Inclusion in PubMed and all major indexing services

- Maximum visibility for your research

Submit your manuscript at www.biomedcentral.com/submit
) Biomed Central 